\title{
TB and M/XDR-TB infection control in European TB reference centres: the Achilles' heel?
}

\section{To the Editors:}

The emergence of multidrug-resistant (MDR) tuberculosis (TB), defined as in vitro resistance to isoniazid and rifampicin, and extensively drug-resistant (XDR)-TB, defined as in vitro drug resistance to isoniazid and rifampicin plus any fluoroquinolone and at least one of the injectable drugs (amikacin, capreomycin or kanamycin), represents a major threat to TB control at the global level [1-5]. XDR-TB is a manmade product, resulting, in essence, from clinical mismanagement of newly diagnosed susceptible and resistant TB cases [2-5]. Moreover, existing MDR/XDR-TB cases must be promptly detected and treated, and the transmission from infectious source cases has to be prevented, particularly in nosocomial settings [2-6].

The World Health Organization (WHO), in its Stop TB Strategy [7] and its recent Policy on Infection Control [8], clearly underlines the importance of implementing effective measures of infection control in clinical facilities managing TB and MDRTB patients. At present, no international study is available to investigate how infection control measures are implemented in healthcare facilities managing TB patients.

The aim of the present study was to document how infection control measures are implemented in selected settings in the European Union, focusing on national MDR/XDR-TB reference centres.

A standardised and comprehensive survey tool was developed, as discussed previously [9, 10]. In summary, the tool consisted of items covering the key areas that a panel of experts considered adequate to evaluate TB infection control in the selected countries. A Delphi process was used to identify the key elements belonging to infection control, and to assign priorities to the questions proposed and define a weighted score to the answers obtained. The items relate to individual patients (risk factors for MDR-TB) and institutional factors (table 1).

The final version of the tool was composed of three parts and one annex: part 1 summarised the features of the setting surveyed (62 items); part 2 collected information on individual clinical records surveyed (one line per clinical case; 141 items); and part 3 allowed comparison of the key case management decisions taken on each individual case against internationally agreed standards (25 scored questions). The international reference standards used to define the highest-standard practice for comparison were derived from the WHO Policy on Infection Control [8] and the International Standards for Tuberculosis Care document [11].

The tool was applied to each individual original patient record examined, and adherence/nonadherence entered on the electronic data collection sheet and, finally, a score. The survey teams, including clinical and public health experts, were trained to use the tool, define adherence/nonadherence to standards and assign the score.

The original records of $40 \mathrm{~TB} / \mathrm{MDR}-\mathrm{TB}$ cases were assessed at each study site, including 30 MDR-TB and 10 other than MDRTB cases (i.e. the strain being susceptible, monoresistant or polyresistant), between November 2009 and March 2010. The clinical files of the cases to survey were selected by the study site counterparts before the visit, following a time criterion (cases treated since January 1, 2005) for a simple random sampling.

The TB reference institutions, identified in collaboration with national health authorities (ministries of health), were identified in five countries illustrative of the different TB epidemiology patterns in Europe (table 1). As per ECDC policy, the selected countries cannot be disclosed.

Categorical variables were compared by Chi-squared or Fisher's exact tests where appropriate. A p-value of $\leqslant 0.05$ was deemed statistically significant. Data were analysed using Stata 11.0 (StataCorp, College Station, TX, USA).

The main findings are described in table 1.40 medical records were assessed per country.

Individual risk factors were generally well documented: investigation of previous TB diagnosis was performed in 186 (93\%) out of $200 \mathrm{~TB}$ cases admitted to the reference centres, although only $160(80 \%)$ out of 200 of their contacts were investigated, which is potentially relevant from a public health perspective.

Although healthcare workers were sufficiently protected in the majority of cases (199 (99.5\%) out of 200 cases) and respirators were always available (200 (100\%) out of 200 cases), 40 (20\%) out of 200 cases were not educated on cough etiquette and other important infection control issues.

No negative-pressure room (including a ventilation system designed so that air flows from the corridors, or any adjacent area, into the negative-pressure room, ensuring that contaminated air cannot escape from the negative-pressure room to other parts of the facility) was identified in four out of five surveyed centres.

Furthermore, specialised training in infection control and healthcare epidemiology was not offered to the individuals employed in the centres surveyed by professional organisations or scientific societies, and respirator fitting was never tested. No specific programme for the implementation of good infection control practice was in place. A surveillance system for monitoring mycobacterial drug resistance covered all cases, although an infection control committee was in place at the time of admission for 160 (80\%) out of 200 cases.

The reference centre located in the group 5 country exemplified the main shortcomings: the proportions scored on 
TABLE 1 Infection control implementation in tuberculosis (TB) reference centres of five European countries, 2009-2010

\begin{tabular}{|c|c|c|c|c|c|c|c|}
\hline Infection control areas & $\begin{array}{c}\text { European } \\
\text { cohort }\end{array}$ & $\begin{array}{l}\text { Group } 1 \\
\text { country }\end{array}$ & $\begin{array}{l}\text { Group } 2 \\
\text { country }\end{array}$ & $\begin{array}{l}\text { Group } 3 \\
\text { country }\end{array}$ & $\begin{array}{l}\text { Group } 4 \\
\text { country }\end{array}$ & $\begin{array}{l}\text { Group } 5 \\
\text { country }\end{array}$ & p-value \\
\hline Investigation of previous TB diagnosis performed & 186/200 (93) & $40(100)$ & $40(100)$ & $40(100)$ & $40(100)$ & $26(65)$ & $<0.001$ \\
\hline Investigation of contacts performed & $160 / 200(80)$ & $40(100)$ & $40(100)$ & $40(100)$ & $40(100)$ & $0(0)$ & $<0.001$ \\
\hline Diagnostic algorithm correctly applied & $189 / 200(94.5)$ & 39 (97.5) & $40(100)$ & $40(100)$ & $38(95)$ & $32(80)$ & 0.001 \\
\hline Administrative measures adequate and implemented & 193/200 (96.5) & $38(95)$ & $40(100)$ & $40(100)$ & $39(97.5)$ & $36(90)$ & 0.103 \\
\hline Surveillance system for drug resistance implemented & $200 / 200(100)$ & $40(100)$ & $40(100)$ & $40(100)$ & $40(100)$ & $40(100)$ & \\
\hline Staff personal protective measures implemented & 199/200 (99.5) & $40(100)$ & $39(97.5)$ & $40(100)$ & $40(100)$ & $40(100)$ & \\
\hline Cough etiquette implemented & $160 / 200(80)$ & $40(100)$ & $40(100)$ & $40(100)$ & $40(100)$ & $0(0)$ & $<0.001$ \\
\hline Respirators and other personal protection equipment available & $200 / 200(100)$ & $40(100)$ & $40(100)$ & $40(100)$ & $40(100)$ & $40(100)$ & \\
\hline Training of staff in infection control performed & $160 / 200(80)$ & $40(100)$ & $40(100)$ & $40(100)$ & $40(100)$ & $0(0)$ & $<0.001$ \\
\hline
\end{tabular}

Data are presented as $n(\%)$, unless otherwise stated. Groups 1-5 represent countries (not necessarily in this order) in the former Soviet Union with an intermediate incidence of TB (two groups), in northern Europe with a low incidence of TB, in southern Europe with a low incidence of TB, and in southern Europe with an intermediate incidence of TB (one group each).

investigation of previous TB diagnosis, contact investigation, infection control committee, cough etiquette and staff training were significantly lower $(p<0.05)$ than those in the other countries. Environmental measures (i.e. methods used to decrease the quantity of droplet nuclei and to control their direction in the air) were inadequate in group 2 and 5 countries $(\mathrm{p}<0.001)$.

The main area of concern was the lack of a comprehensive infection control strategy or plan in all the centres surveyed, reflecting a lack of central-level planning [9]. Administrative measures (infection control committees) were not systematically in place. A plan is a key instrument to activating and empowering the necessary committees, and ensuring that the key infection control activities are implemented and evaluated. The pitfalls identified in the areas of training and health education would benefit from the implementation of infection control plans at the facility level. The lack of planning at the facility level reflects a lack of planning at the central level on this given issue.

In terms of personal protection, although respirators were in place and used by staff, and surgical masks by patients, respirator fit was not tested in any of the centres. It is known that a proportion of staff do not pass respirator fit testing, due to inadequate positioning of the respirator [12]. A respirator placed wrongly on the mouth and nose offers a false sense of security, and a lack of real protection from Mycobacterium tuberculosis infection cannot be excluded [9, 12].

The lack of negative-pressure ventilation in all but one of the centres surveyed deserves a special discussion. Sufficient respiratory isolation rooms for all new patients admitted (at least until the exact resistance pattern is identified and/or the patient is rendered noninfectious) and adequate isolation procedures need to be available in centres diagnosing and treating TB. Although a lack of negative-pressure ventilation rooms, even for MDR-TB cases, has been identified, no evidence was found that this caused secondary cases among healthcare staff (e.g. a single ТВ case was identified in one country over a 10-yr period as a result of this survey). A critical review of the available evidence and clear recommendations on this procedure seem to be justified.

According to the results of this survey, administrative and personal protection measures (e.g. masks for patients or respirators for healthcare staff) should be scaled up, as they are relevant in preventing secondary TB cases among staff. Furthermore, adequate health education practices for patients (e.g. cough etiquette) coupled with regular training of staff are necessary to ensure good infection control results.

The results of our study clearly demonstrate that even in European MDR/XDR-TB reference centres, infection control measures do not fully comply with international recommendations and margins exist for prompt public health action aiming at minimising TB transmission in nosocomial settings.

\section{G. Sotgiu*, L. D'Ambrosio", R. Centis", G. Bothamley",} D.M. Cirillo ${ }^{+}$, S. De Lorenzo ${ }^{\S}$, G. Guenther ${ }^{f}$, K. Kliiman** R. Muetterlein"\#, V. Spinu" "थ , M. Villar ${ }^{++}$, J.P. Zellweger ${ }^{\S \S}$, A. Sandgren ${ }^{f f}$, E. Huitric ${ }^{f f}$, C. Lange ${ }^{f}$, D. Manissero ${ }^{f f}$ and G.B. Migliori"

${ }^{*}$ Hygiene and Preventive Medicine Institute, University of Sassari, Sassari, "WHO Collaborating Centre for TB and Lung Diseases, Fondazione S. Maugeri, Care and Research Institute, Tradate, ${ }^{+}$Emerging Bacterial Pathogens Unit, San Raffaele Scientific Institute, Milan, and ${ }^{\S} \mathrm{E}$. Morelli Hospital, Reference Hospital for MDR and HIV TB, Sondalo, Italy. "Homerton University Hospital, London, UK. ${ }^{f}$ Division of Clinical Infectious Diseases, Medical Clinic, Research Centre Borstel, Borstel, and "\# Dept of Medicine, Parsberg Hospital, Parsberg, Germany. **Dept of Pulmonary Medicine, University of Tartu, Tartu, Estonia. ${ }^{~}{ }^{\top}$ MDR-TB Centre, Marius Nasta Institute of Pulmonology, Bucharest, Romania. ${ }^{++}$General Directorate of 
Health in Lisbon and Lung Diseases Centre of Venda Nova, Amadora, Portugal. ${ }^{\S \S}$ TB Clinic, Dept of Ambulatory Care and Community Medicine, University of Lausanne, Lausanne, Switzerland. ${ }^{f f}$ Scientific Advice Unit, European Centre for Disease Prevention and Control, Stockholm, Sweden.

Correspondence: G.B. Migliori, World Health Organization Collaborating Centre for Tuberculosis and Lung Diseases, Fondazione S. Maugeri, Care and Research Institute, Via Roncaccio 16, 21049, Tradate, Italy. E-mail: giovannibattista. migliori@fsm.it

Support Statement: The research leading to this publication has been performed under the European Centre for Disease Prevention and Control (ECDC) service contract 1757 adjudicated to TBNET following open tender competition.

Statement of Interest: None declared.

\section{REFERENCES}

1 World Health Organization. Multidrug and Extensively DrugResistant TB (M/XDR-TB). 2010 Global Report on Surveillance and Response. WHO/HTM/TB/2010.3. Geneva, World Health Organization, 2010.

2 Sotgiu G, Ferrara G, Matteelli A, et al. Epidemiology and clinical management of XDR-TB: a systematic review by TBNET. Eur Respir J 2009; 33: 871-881.

3 Migliori GB, Loddenkemper R, Blasi F, et al. 125 years after Robert Koch's discovery of the tubercle bacillus - the new XDR-TB threat.
Is "science" enough to tackle the epidemic? Eur Respir J 2007; 29 423-427.

4 Migliori GB, Besozzi G, Girardi E, et al. Clinical and operational value of the extensively drug-resistant tuberculosis definition. Eur Respir J 2007; 30: 623-626.

5 Migliori GB, Ortmann J, Girardi E, et al. Extensively drug-resistant tuberculosis, Italy and Germany. Emerg Infect Dis 2007; 13: 780-782.

6 World Health Organization. Extensively drug-resistant tuberculosis (XDR-TB): recommendations for prevention and control. Wkly Epidemiol Rec 2006; 81: 430-432.

7 Raviglione MC, Uplekar MW. WHO's new Stop TB Strategy. Lancet 2006; 367: 952-955.

8 World Health Organization. WHO Policy on Infection Control in Health-Care Facilities, Congregate Settings and Households. WHO/HTM/TB/2009.419. Geneva, World Health Organization, 2009.

9 Sotgiu G, Centis R, D'Ambrosio L, et al. Development of a standardised tool to survey MDR-/XDR-TB case management in Europe. Eur Respir J 2010; 36: 208-211.

10 Miravitlles M, Ferrara G, Lange C, et al. TB or not TB: update from the ERS Respiratory Infection Assembly 10. Eur Respir J 2010; 36: 665-670.

11 Tuberculosis Coalition for Technical Assistance. International Standards for Tuberculosis Care (ISTC), 2nd Edn. The Hague, Tuberculosis Coalition for Technical Assistance, 2009.

12 Nardell E, Dharmadhikari A. Turning off the spigot: reducing drug-resistant tuberculosis transmission in resource-limited settings. Int J Tuberc Lung Dis 2010; 14: 1233-1243.

\section{Effects of distance to treatment centre and case load upon tuberculosis treatment completion}

\section{To the Editors:}

The prompt identification and adequate treatment of tuberculosis (TB) cases are key components of the global control effort [1]. In many high-income countries, TB is relatively uncommon, implying that many clinicians do not have regular and continuing experience of managing TB. To address this, a policy response is a trend towards small numbers of centralised treatment facilities where individuals may receive better and more complete treatment $[2,3]$. This centralising trend is the opposite to many low-income countries, where there is a move towards larger numbers of more decentralised TB services to facilitate patient access [4]. The difference is that such countries have sufficient cases to allow clinical staff to maintain their expertise, even in rural areas.

If $\mathrm{TB}$ services become more centralised, then patients may experience greater difficulty in accessing $\mathrm{TB}$ services due to increasing the distance between the home and treatment centre. There is little research on how distance affects TB completion, but rural residence is a known risk factor associated with a delay in the diagnosis and treatment of TB
[5] and may therefore affect treatment completion. However, most of this evidence comes from low-income countries. In contrast, in high-income countries, good transport links and reasonable social security systems imply that such barriers may not exist.

This epidemiological study examined whether treatment centre case load (annual number of TB patients seen) and healthcare accessibility (proximity of patient residence to TB treatment centre) have an influence upon the completion of TB treatment in England and Wales, UK.

The study was based upon a sample of 21,954 patients reported to the national enhanced TB surveillance system (ETS) from 2001 to 2006 in England and Wales. These TB patients all had their diagnosis confirmed by bacteriological culture or had clinical/radiological/histopathological features suggestive of $\mathrm{TB}$, and their clinician had taken the decision to treat the patient with a full course of anti-TB therapy. This sample was $50.3 \%$ of the total cases reported to the ETS during this period. Cases had to be excluded from the study mainly due to missing information on where the patient was actually treated 